\title{
Whole-blood metabolomics of dementia patients reveal classes of disease-linked metabolites
}

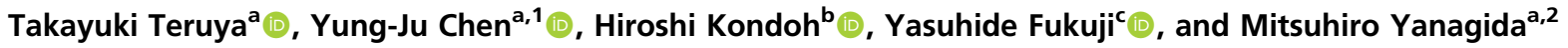 \\ ${ }^{a}$ G0 Cell Unit, Okinawa Institute of Science and Technology Graduate University, Okinawa 904-0495, Japan; ${ }^{b}$ Geriatric Unit, Graduate School of Medicine, \\ Kyoto University, Kyoto 606-8507, Japan; and 'National Hospital Organization Ryukyu Hospital, Okinawa 904-1201, Japan
}

Contributed by Mitsuhiro Yanagida, May 14, 2021 (sent for review November 3, 2020; reviewed by Hidenori Arai and Barry Halliwell)

Dementia is caused by factors that damage neurons. We quantified small molecular markers in whole blood of dementia patients, using nontargeted liquid chromatography-mass spectroscopy (LC-MS). Thirty-three metabolites, classified into five groups (A to E), differed significantly in dementia patients, compared with healthy elderly subjects. Seven A metabolites present in plasma, including quinolinic acid, kynurenine, and indoxyl-sulfate, increased. Possibly they act as neurotoxins in the central nervous system (CNS). The remaining 26 compounds ( $B$ to $E$ ) decreased, possibly causing a loss of support or protection of the brain in dementia. Six B metabolites, normally enriched in red blood cells (RBCs), all contain trimethylated ammonium moieties. These metabolites include ergothioneine and structurally related compounds that have scarcely been investigated as dementia markers, validating the examination of RBC metabolites. Ergothioneine, a potent antioxidant, is significantly decreased in various cognition-related disorders, such as mild cognitive impairment and frailty. C compounds also include some oxidoreductants and are normally abundant in RBCs (NADP', glutathione, adenosine triphosphate, pantothenate, $S$-adenosyl-methionine, and gluconate). Their decreased levels in dementia patients may also contribute to depressed brain function. Twelve D metabolites contains plasma compounds, such as amino acids, glycerophosphocholine, dodecanoyl-carnitine, and 2-hydroxybutyrate, which normally protect the brain, but their diminution in dementia may reduce that protection. Seven D compounds have been identified previously as dementia markers. B to E compounds may be critical to maintain the CNS by acting directly or indirectly. How RBC metabolites act in the CNS and why they diminish significantly in dementia remain to be determined.

dementia | Alzheimer's disease | antioxidants | ergothioneine | trimethyl-ammonium compounds

$\boldsymbol{\boldsymbol { U }}$ Dementia" is a collective term to describe various symptoms of cognitive impairment in a condition in which intelligence is irreversibly diminished due to acquired organic disorders of the brain, characterized by deterioration of memory, thinking, behavior, and the ability to perform daily activities $(1,2)$. Though a common cause is Alzheimer's disease (AD), a neurodegenerative disease in which memory is rapidly impaired due to hippocampal atrophy, multiple types of dementia, known as "mixed dementia," can coexist $(3,4)$. Mental and physical exercise and avoidance of obesity may reduce the risk of dementia (5-7). No medications or supplements have been definitively shown to decrease risk $(8,9)$. Dementia most often begins in people over $65 \mathrm{y}$ of age, and about $6 \%$ of seniors are afflicted with it. It is one of the most costly diseases in developed countries (10).

In this study, we conducted nontargeted, comprehensive analysis of blood metabolites in dementia patients. Thorough metabolomic evaluation can supply complete information about metabolite abundance in each subject. While nontargeted analysis is far more laborious than targeted analysis, the effort expended in this "no assumptions" approach is often recompensed by identification of diagnostic compounds overlooked by targeted analysis. A wealth of metabolite information may provide clues to understanding the profound metabolic changes occurring in dementia. Liquid chromatography-mass spectroscopy (LC-MS) was employed for whole-blood metabolite profiling of dementia patients, and we found metabolic compounds not previously known to be related to dementia. Metabolomics of blood cells have scarcely been investigated, particularly in relation to diseases, despite the fact that red blood cells (RBCs) account for about $40 \%$ of all blood metabolites (11-13). Thus, metabolomic information from RBCs also provides crucial information on health and disease (14-16). Here we identified 33 dementia-linked markers (12 of which are RBC-enriched) and validated them by principal component analysis (PCA), correlation, and heatmap analyses, confirming that these markers actually are involved in development of dementia. Our results suggest that detailed molecular diagnosis of dementia is now possible. Somewhat unexpectedly, markers deduced from dementia only partially overlap with amino acid markers obtained from frailty patients with cognitive defects (16), so that frailty and dementia partly share the diminished cognitive markers. We also show that an antioxidant, ergothioneine (ET), an RBC component involved in human cognitive ability $(16,17)$, and two related compounds are reduced in dementia.

\section{Significance}

Dementia is a slowly progressing, chronic, and usually irreversible decline in cognitive function. Mechanistic causes and definitive treatments remain elusive. Using comprehensive metabolomics, we identified five groups of 33 metabolites (A to E), 13 of them previously reported, possibly useful for diagnosis and therapy of forms of dementia, such as Alzheimer's disease. Seven A compounds may act as neurotoxins, whereas $B$ to $E$ compounds may protect the nervous system against oxidative stress, maintain energy reserves, supply nutrients and neuroprotective factors. Five metabolites, ergothioneine, $S$-methyl-ergothioneine, trimethyl-histidine, methionine, and tryptophan, overlap with those reported for frailty. Interventions for cognitive diseases involving these dementia metabolomic markers may be accomplished either by inhibiting $A$ compounds or by supplementing B to E compounds in patients.

Author contributions: M.Y. designed research; T.T., Y.-J.C., and M.Y. performed research; Y.F. provided clinical samples and information; T.T., Y.-J.C., H.K., and M.Y. analyzed data; and T.T., Y.-J.C., H.K., and M.Y. wrote the paper.

Reviewers: H.A., National Center for Geriatrics and Gerontology; and B.H., National University of Singapore.

The authors declare no competing interest.

This open access article is distributed under Creative Commons Attribution-NonCommercialNoDerivatives License 4.0 (CC BY-NC-ND).

1Present address: Department of Food Science, Tunghai University, Taichung 407224, Taiwan, People's Republic of China.

${ }^{2}$ To whom correspondence may be addressed. Email: myanagid@gmail.com.

This article contains supporting information online at https://www.pnas.org/lookup/suppl/ doi:10.1073/pnas.2022857118/-/DCSupplemental.

Published September 7, 2021. 


\section{Results}

Collection of blood samples from dementia subjects. To identify dementia-related blood metabolites, quantitative comparisons were conducted of blood samples of dementia patients and healthy elderly (HE) and healthy young (HY) subjects. Blood samples of dementia patients (age 75 to $88 \mathrm{y}$ ) diagnosed and hospitalized at the National Hospital Organization Ryukyu Hospital, Kin-town, Okinawa were obtained from each patient after informed consent (Materials and Methods). The same numbers of HE (67 to $80 \mathrm{y}$ ) and HY (28 to $34 \mathrm{y}$ ) volunteers from Onna Clinic, Onna-village, Okinawa were also recruited (SI Appendix, Fig. S1 and Table S1). Twenty-four subjects comprising eight dementia patients, eight $\mathrm{HE}$ subjects, and eight $\mathrm{HY}$ subjects participated in this study. All blood samples were drawn at each hospital as described (14). Venous blood samples were taken into tubes with heparin as an anticoagulant.

Thirty-three dementia-linked blood metabolites comprise five subgroups, A to E. In all whole-blood samples collected, 124 metabolites were identified and quantified by nontargeted LC-MS (SI Appendix, Table S2). They consisted of 14 subgroups. Fifty-one compounds, comprising 5 subgroups (nucleotides, vitamins and coenzymes, nucleotides/sugar derivatives, sugar phosphates, and antioxidants) are RBC-enriched (14). Of these 124 compounds, 33 metabolites differed significantly between dementia patients and $\mathrm{HE}$ subjects (range of $P$ values, $0.00016<P<0.05$ ) (Fig. 1 and Table 1). Five compounds, adenosine triphosphate (ATP), glutathione disulfide (GSSG), glutamine, phenylalanine, and betaine, are highly abundant (ranked H). Five other compounds, glycerophosphocholine, ET, methionine, tryptophan, and tyrosine, are of high to medium (H-M) abundance. Three additional compounds vary widely in abundance among healthy subjects (high to low; H-L): caffeine, dimethyl-xanthine, and trimethyl-tryptophan. The remaining 20 compounds are of medium to low abundance (M-L, M, L) (Table 1). Twelve of the 33 compounds are RBC-enriched, which has been scarcely reported. Characteristically, 9 dementia-related compounds contain trimethyl-ammonium moieties (Table 1).

Peak ratios (dementia/HE in Table 1) were calculated using the median of peak abundance in each group. Seven compounds (group A) that increased in dementia showed peak ratios $>1.0$. Twenty-six others had ratios $<1.0$ that declined in dementia. Four compounds exhibiting the greatest decreases are caffeine-related: dimethyl-xanthine (E; 0.04), trimethyl-tyrosine (B; 0.08), caffeine (E; 0.09), and trimethyl-tryptophan (B; 0.10). Curiously, all are aromatics. Their variances are also broad in healthy subjects.

To quantify individual variability of the 124 metabolites, coefficients of variation (CVs) for all experimental populations of the 24 subjects were calculated (SI Appendix, Table S2). In the 33 dementia-linked compounds, CVs of ATP (0.20), GSSG (0.14), and $\mathrm{NADP}^{+}(0.33)$, which have vital functions, were relatively low, while trimethyl-tryptophan (1.09), trimethyl-tyrosine (2.21), trimethyl-phenylalanine (3.17), and caffeine (1.49) were high. These values were substantially in agreement with those in our previous study, an independent dataset obtained from $30 \mathrm{HE}$ and HY subjects (14). Thus, the great variability of data in Fig. 1 reflects genuine individual variation in metabolites, which were accurately detected by our metabolomic analysis. These data demonstrate that compounds having small to large individual variability are implicated in dementia.

Increased group A compounds may be toxic. Seven group A compounds were identified by their increases in the dementia patients compared to HE (Fig. 1A). They consisted of indoxyl-sulfate (peak ratio dementia/HE, 1.93), quinolinic acid (1.79), adenosine (1.51), dimethyl-guanosine (1.45), N6-acetyl-lysine (1.47), pseudouridine (1.19), and kynurenine (1.12). Two of the seven metabolites were previously reported as AD-related markers $(18,19)$. Some of them are reportedly toxic (20), suggesting that they may be inhibitory in the brain (see below). To determine the statistically difference, the non-parametric Mann-Whitney $U$ test was used. This test examines the significant dirrefence between groups based on ranks and not values. Since ranks represent the relative position of an individual in comparison to others, it is not affected by outlier value.

Twenty-six blood dementia compounds consist of four groups, B to E. The 26 remaining compounds decreased in dementia patients $(P<$ 0.05 ) (Fig. $1 B-E$ ). They consisted of four subgroups (B to E), having distinct characteristics. Group B compounds include ET and five other trimethyl-ammonium compounds. To our knowledge, except for ET (17), these are all not previously reported as dementia markers, probably because they are enriched in RBCs and scarcely studied in connection with dementia. ET is an antioxidant, a thiourea derivative of trimethyl-histidine. Two other ET-related, but less abundant, compounds, $S$-methyl-ET and trimethyl-histidine (hercynine), also declined strikingly in blood of dementia patients.

Group C compounds also decreased in dementia patients. These included ATP, NADP ${ }^{+}$(oxidoreductive coenzyme), GSSG (redox compound), pantothenate (vitamin B5), $S$-adenosyl-methionine (SAM; methyl donor) (21), and gluconate (zinc carrier) (22). They are related to energy, redox reactions, methylation, and metal ions. Group C compounds were all enriched in RBCs, and four of the six are not previously reported as dementia markers. Two of them (SAM and GSSG) were previously shown to be ADrelated $(21,23)$.

Trimethyl-tryptophan (hypaphorine), trimethyl-phenylalanine, glycerophosphocholine, dodecanoyl-carnitine (24), and trimethyltyrosine, all of which contain trimethyl-ammonium ions, also declined. The extent of reduction for trimethyl-tryptophan (0.10) and trimethyl-tyrosine $(0.08)$ was striking. These reductions may be due to instability or reduced synthesis, or to reduced import in dementia patients. Of the nine compounds that contain a trimethylammonium moiety, six of them that contain ET are enriched in RBCs and classified as group B compounds (Table 1).

Twelve group D metabolites (Table 1) are enriched in blood plasma and seven of them were previously reported to be dementia or AD markers. They include standard amino acids, glutamine $(19,25)$, phenylalanine $(19,26)$, tyrosine (19), histidine $(19,25)$, methionine, and tryptophan (regular amino acids) (18, 19), a pyrimidine nucleoside, uridine (27), and organic acids, 2-hydroxybutyrate (lipid-degradation product) and keto(iso)leucine (keto acid). Caffeine is a known dementia marker (28). Dimethyl-xanthine is a metabolite of caffeine. These greatly declined in dementia and are highly correlated with and isolated from other metabolites (see below) so they are designated as group E. Consistency of group D plasma metabolites as dementia markers but not group $\mathrm{B}$ and $\mathrm{C} \mathrm{RBC}$ metabolites validated the method of searching dementia markers that we employed in the present study. The great majority of metabolites enriched in RBCs were not identified in the previous studies.

Nine trimethylated ammonium compounds were diminished in dementia patients. Of nine trimethylated compounds that decreased in dementia, six are enriched in RBCs (SI Appendix, Fig. S2). Three of them (betaine, glycerophosphocholine, and dodecanoyl-carnitine) are present in plasma and are synthesized in the human body, whereas the other six, containing an aromatic moiety, are derived from food $(29,30)$. Most strikingly, six of these nine compounds are highly abundant $(\mathrm{H}, \mathrm{H}-\mathrm{M}$, or $\mathrm{H}-\mathrm{L})$ in plasma and RBCs in healthy subjects, and are highly correlated so that their behavior may be highly coordinated. Hence, the sharp declines of these amphipathic compounds (possessing both hydrophilic and lipophilic properties and forming the basis of lipid polymorphism) in blood of dementia patients may strongly affect the physicochemical properties of neuronal systems. 

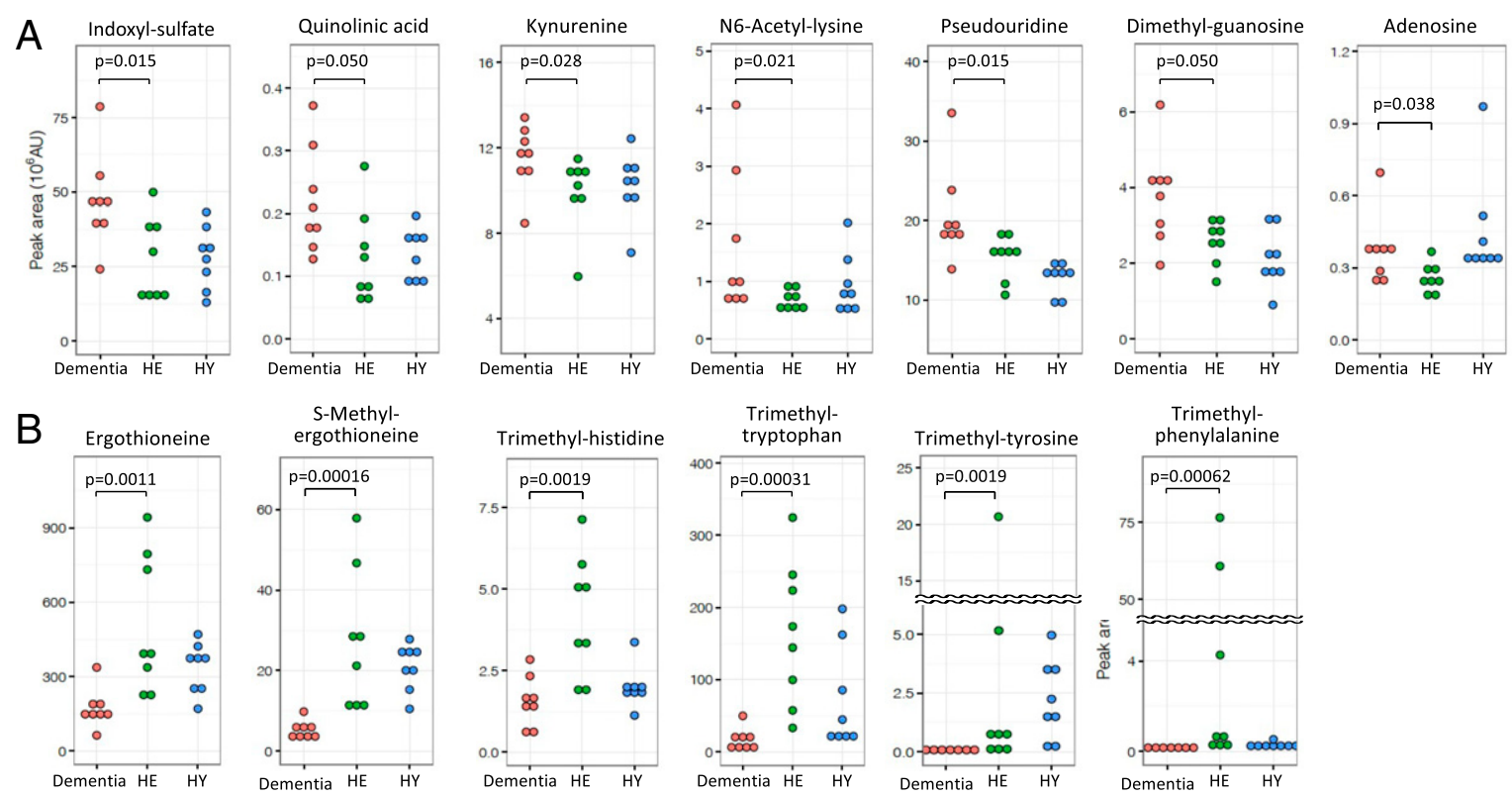

C

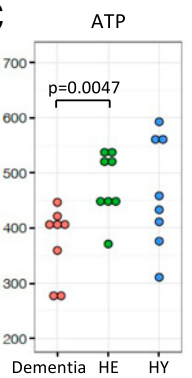

S-Adenosyl-methionine
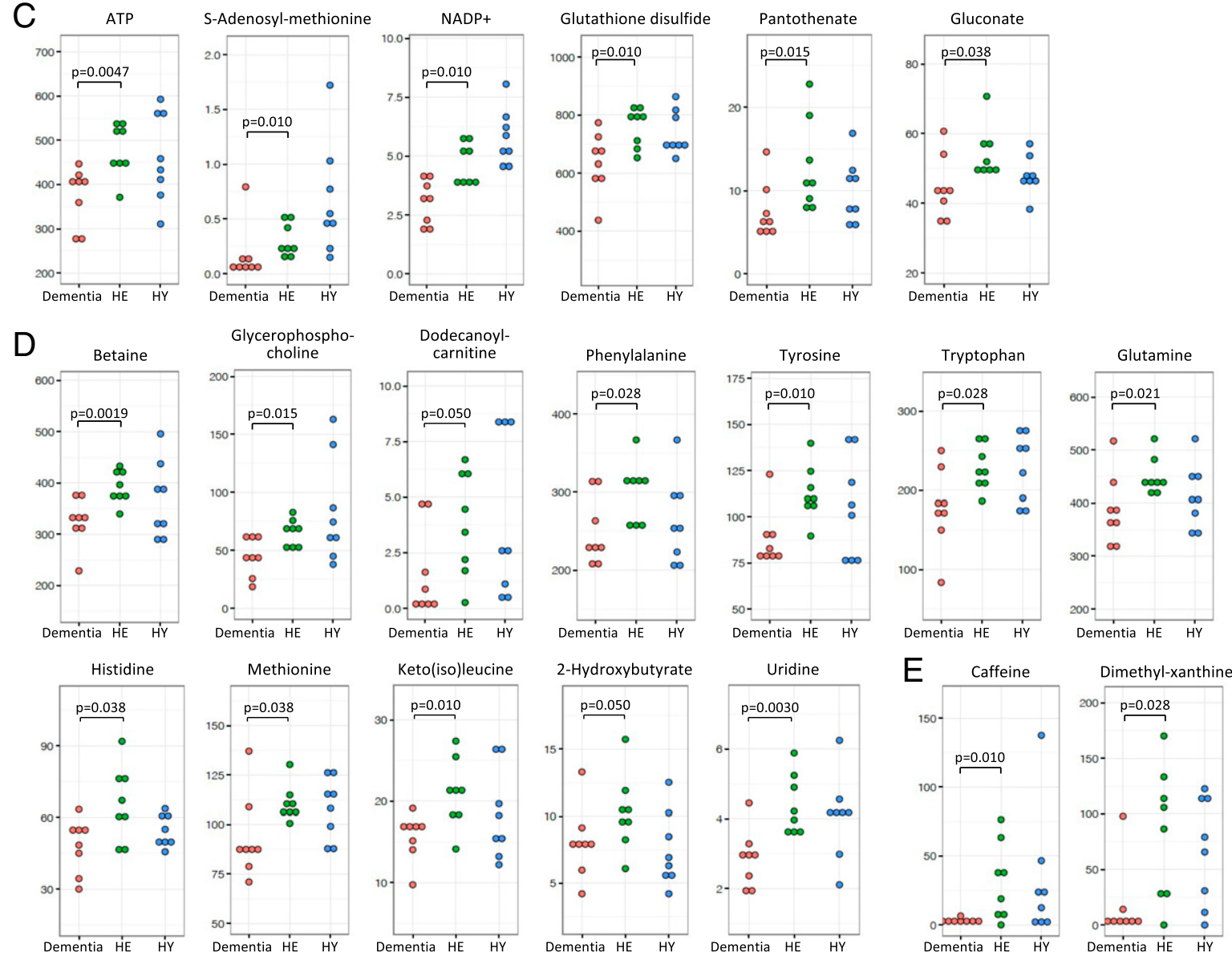

Fig. 1. Dot plot profiles of 33 dementia-related metabolites. Dementia metabolites were selected using $P$ values $(<0.05)$ obtained by comparison of peak abundance between dementia and HE subjects. Compounds were grouped into A to E (see text).

Seven metabolites increased in dementia. Interestingly, the seven metabolites of group $\mathrm{A}$, comprising three nucleosides and four amino acid derivatives, increased in dementia (Fig. $1 A$ ). None was highly abundant and none was enriched in RBCs, and their increase in dementia occurred in plasma. Indoxyl-sulfate, kynurenine, and quinolinic acid (18) are involved in tryptophan metabolism and possibly act as excitatory toxins in the brain (31, 32 ), while N6-acetyl-lysine is implicated in histone and nonhistone protein modification (33). Pseudouridine, adenosine (19), and dimethyl-guanosine are degradation products of RNAs present in urine and are thought to be oxidized $(34,35)$. Increases of these metabolites in dementia are of great interest, as 
Table 1. List of 33 dementia markers

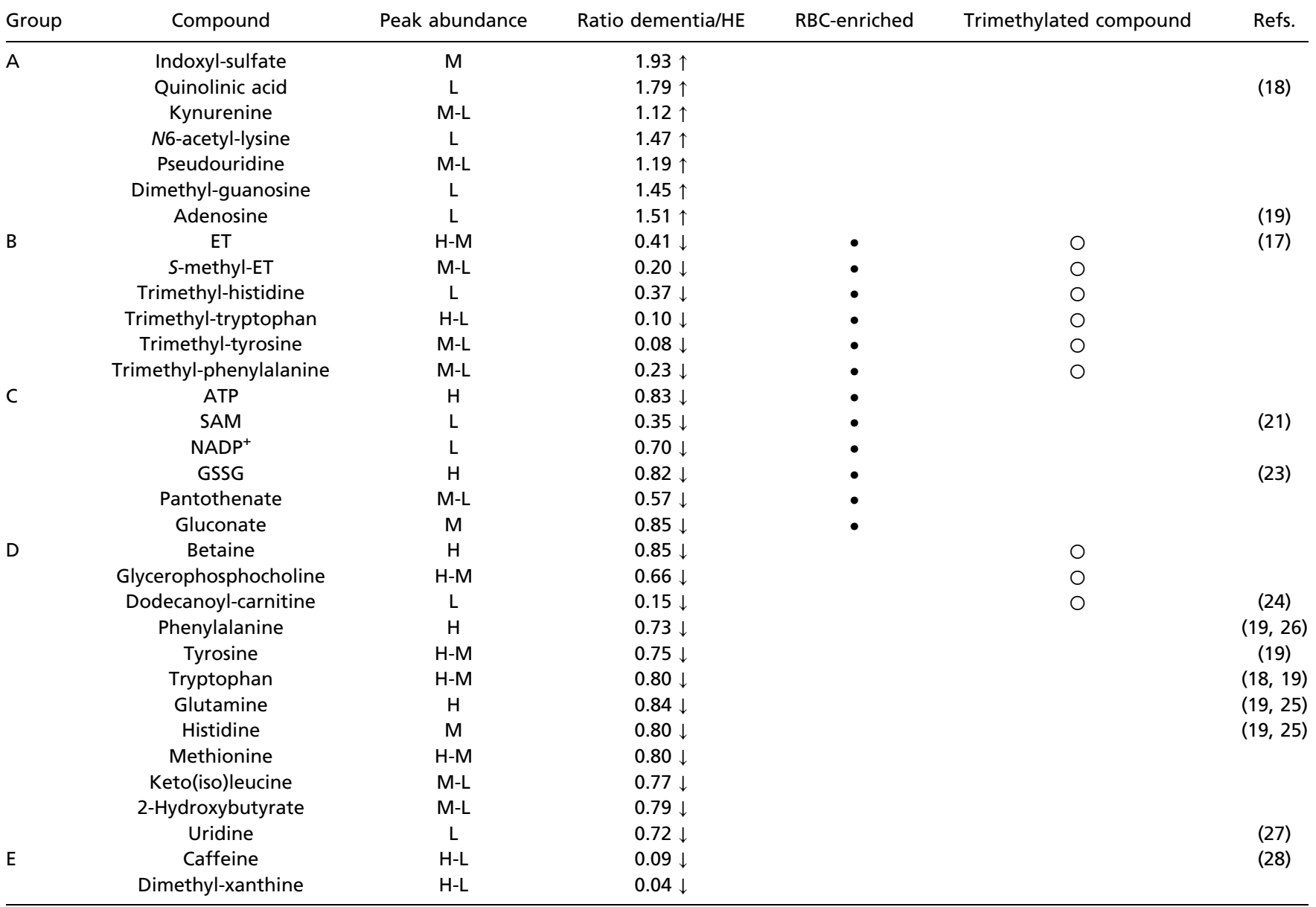

Thirty-three compounds showed significant differences in abundance between dementia patients and HE subjects. Abundances of compounds (peak areas) are displayed. The peak ratio was calculated using the median of peak abundance in dementia and HE subjects, respectively. Twelve RBC-enriched compounds and nine trimethylated compounds are shown with closed and open circles, respectively. The references for dementia markers previously reported are listed.

some are reportedly toxic in the central nervous system (CNS) and may lead to impairment of the brain (36-38).

PCA separates dementia patients from nondementia subjects. To distinguish between dementia and $\mathrm{HE}$ subjects, we then applied PCA. We calculated PC (PC1, PC2) values using abundance data of the 33 dementia-related metabolites in the 16 subjects. Dementia and HE subjects were clearly separated (Fig. $2 A$ ). We then attempted to achieve the same degree of resolution using fewer metabolites and found that six metabolites (dimethyl-guanosine, pseudouridine, $\mathrm{NADP}^{+}$, trimethyl-histidine, ET, and $S$-methylET) were still able to separate dementia from nondementia subjects almost perfectly (Fig. $2 B$ ).

Correlation analysis corroborates five metabolite groups. Levels of some blood metabolites linked in a biochemical pathway and/or function show correlations (14). We first confirmed correlations within ET derivatives (ET, $S$-methyl-ET, and trimethyl-histidine) and between caffeine and dimethyl-xanthine using Pearson's correlation coefficient $r$. Abundance of these compounds indicated high correlations $(0.92>r>0.76, P<0.0001)$ (SI Appendix, Fig. S3) as previously reported (14). Thus, the dataset of the present study is reproducible and statistically valid.

Second, to gain insight into how these 33 dementia metabolites are related, we searched for relationships among them. Significant positive and negative correlations $(0.89>r>0.50$ or $-0.68<$ $r<-0.50)$ in groups A to E support the classification of the subgroups shown in Fig. 3.

Five of the seven group A markers were correlated (0.89 to 0.57 ) within the subgroup (quinolinic acid, dimethyl-guanosine, pseudouridine, indoxyl-sulfate, and kynurenine). This striking correlation strongly supported their classification within group A and opposition to groups B to E. How group A compounds molecularly oppose groups B to D (Fig. 3, green color) remains to be determined. It is unknown whether the mode of interaction is indirect or direct and remains to be studied.

Negative correlations exist not only between group A and B compounds but also between group A and $\mathrm{C}$ compounds. Group $\mathrm{B}$ and $\mathrm{C}$ metabolites are correlated within the groups, again validating their subgroup designations. Group B metabolites are structurally related, as they commonly contain a trimethylated ammonium group, indicating that they are antioxidative. Group C metabolites are not structurally related, but three of them $\left(\mathrm{NADP}^{+}, \mathrm{GSSG}\right.$, and ATP), implicated in redox and energy metabolism, are highly correlated and enriched in RBCs. These distinct metabolites may coordinate to support directly or indirectly their metabolite pathways. Finally, two metabolites, caffeine and dimethyl-xanthine, in group E were highly correlated; the latter is the precursor of caffeine. There are reports on caffeine showing that these purines are beneficial to relieve dementia (Discussion). 

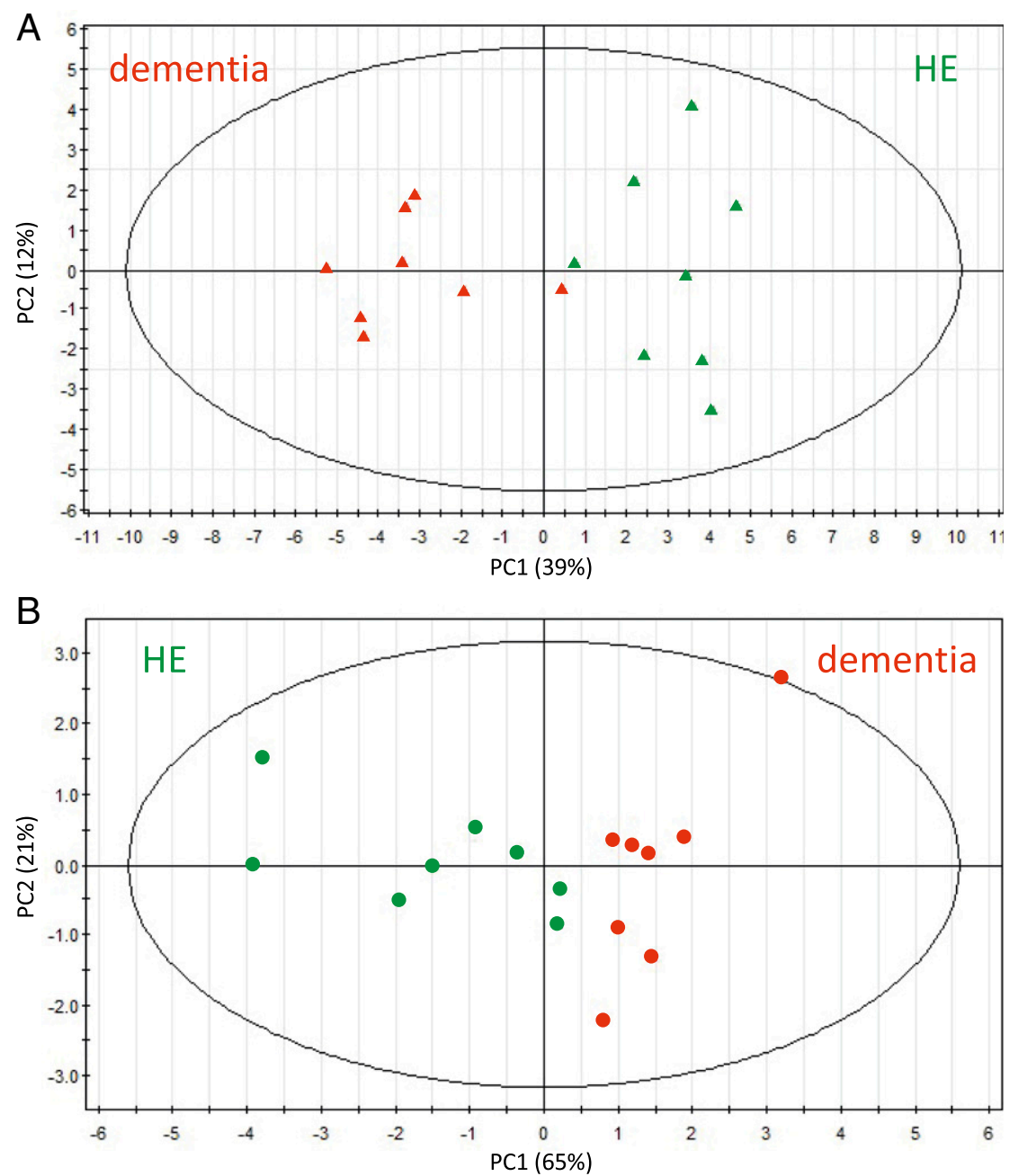

Fig. 2. PCA of 33 AD or 6 selected dementia-related compounds showed significant differences between patients with dementia and HE subjects. Blood data of dementia and HE subjects were subjected to PCA. (A) A PCA plot using abundances of 33 (7 increased and 26 decreased) dementia-related compounds. Dementia and HE subjects were separated into two domains (see text). Red, dementia; green, HE. (B) PCA was also performed using only 6 selected dementia markers (dimethyl-guanosine, pseudouridine, S-methyl-ET, ET, trimethyl-histidine, and NADP ${ }^{+}$).

Heatmap comparison of dementia and HE subjects. Using abundance data of 33 dementia compounds in eight dementia patients and eight HE subjects, a heatmap was constructed (Fig. 4 and SI Appendix, Fig. S4). In dementia patients, the 7 group A compounds mostly displayed red color cells in dementia patients due to the increased abundances, whereas $\mathrm{HE}$ subjects mostly showed blue color cells due to the decreased abundances. In the remaining 26 compounds, dementia subjects displayed blue cells, due to their decrease, whereas HE subjects showed red cells, due to their increase, more than the average. Strikingly, the seven group A compounds increased nearly uniformly in dementia patients, whereas in HE and also HY (SI Appendix, Fig. S4) subjects, the levels were mostly below 50 , indicating that these may be appropriate as diagnostic markers for dementia. Thus, the heatmap profiles showed individual variations of dementialinked metabolites, and further study will provide more evidence for their use as a diagnostic tool.

\section{Discussion}

A principal conclusion of this study is that plasma-enriched dementia factors, group A compounds, increased in dementia patients and might have a negative toxic impact on CNS functions by themselves or their degradation products (SI Appendix, Fig. S5) $(20,32,39-42)$. Other group B to E metabolites may be beneficial for the CNS, as their quantity all declined in dementia patients. RBC-enriched group B metabolites all containing the trimethyl-ammonium ion may protect the CNS through their antioxidative and other unknown activity. Group $\mathrm{C}$ compounds, also RBC-enriched, have cellular functions implicated in energy, redox, and so forth, and may be important for maintaining CNS brain functions. Two compounds of group C, SAM and GSSG, had been reported to be AD- and dementia-related markers (21, 23). Group D contains 12 plasma compounds, half of which had been reported as AD-related markers. We thus speculate the 7 group A compounds pathologically enhance or lead to severe dementia such as AD. This presumed dementia deterioration by group A factors is opposed if group B to E metabolites are sufficiently supplied.

Correlation analysis allowed us to categorize 33 dementia markers into five subgroups (A to E) (Fig. 3). Seven group A compounds seem to be opposed by 26 group B to E compounds. Group A compounds were oxidized, and 5 of them are highly correlated, perhaps due to common relation to tryptophan degradation and/or nucleoside metabolism. Group A compounds may act as inhibitors, poisons, or wastes $(20,39)$. Kynurenine, quinolinic acid (40), and indoxyl-sulfate, known as toxins, may target the brain and worsen dementia (32). N6-acetyl-lysine may be related to the accumulation of acetylated tau and alpha-tubulin in the AD 


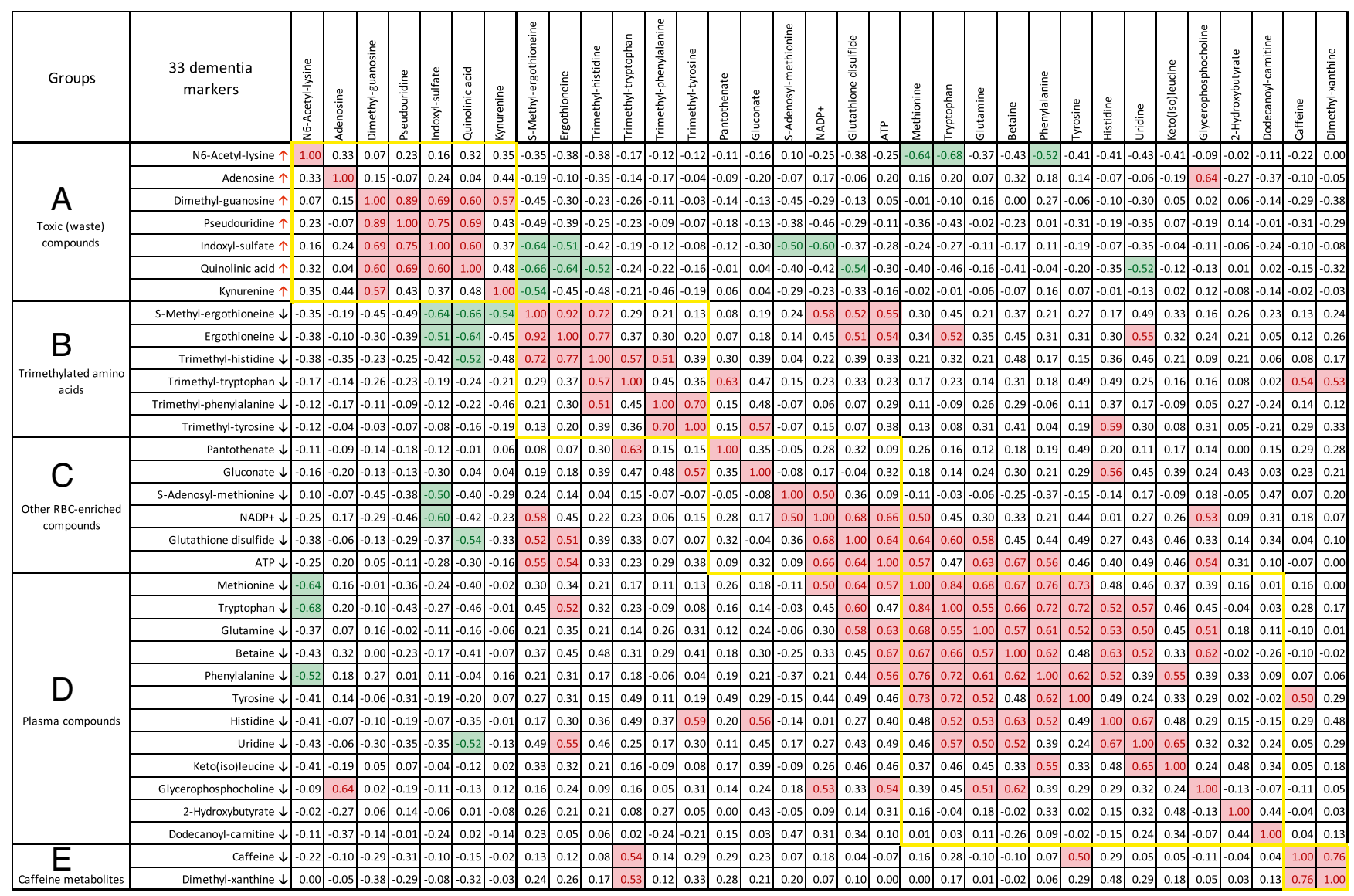

Fig. 3. Correlation analysis of 33 dementia markers. Correlation values $(r)$ larger than +0.5 are marked with red, whereas values below -0.5 are in green Compounds that increased or decreased in dementia patients are indicated by arrows. Five subgroups of 33 compounds are shown with frames in yellow.

brain $(41,42)$. Note, however, that the seven group A markers increased in dementia were not found in frail subjects (16). If the change in group $\mathrm{A}$ is the causal reason for dementia, then a cognitive cause in frailty may be distinct from that of dementia. There seemed to be only a small overlap between dementia- and frailty-related cognition markers in the outcome of patients' metabolite analysis. Five markers are common: They are ET, $S$-methyl-ET, trimethyl-histidine, methionine, and tryptophan. While aging is thought to be a major risk factor for dementia, we have not found clear age markers among dementia factors except these five.

Six group B compounds contained trimethyl-ammonium moieties, presumably acting as antioxidants, so group B may act in opposition to group A, acting in concert to exacerbate dementia when the levels of group B are declined. Cheah et al. indicated that a subject group with mild cognitive impairment (MCI) showed lower ET levels (17). They showed that the whole-blood ET level of MCI subjects was around 0.65 -fold compared with normal subjects. Kameda et al. reported that frail patients showing MCI also showed a decline of ET, $S$-methyl-ET, trimethyl-histidine, tryptophan, and methionine (16). In this study, the levels of ET (0.41), $S$-methyl-ET (0.20), and the other trimethylated ammonium compounds (0.08 to 0.85 ) were also reduced (SI Appendix, Table $\mathrm{S} 3)$. As ET is abundant, for example, in mushrooms, an intervention study will be possible.

Six group $\mathrm{C}$ metabolites also enriched in RBCs include GSSG, $\mathrm{NADP}^{+}$, pantothenate, SAM, ATP, and gluconate, a metal (zinc) carrier. Their actions may directly or indirectly resist dementia, and thus their decline would contribute to it. Glutathione (GSH) is easily oxidized to form inactive GSSG. In this study, we only detected GSSG, as GSH was difficult to stably measure. Zinc, one of the most abundant trace metals in the brain, works for dementia both positively and negatively $(43,44)$. Disruption of zinc homeostasis in the brain is thought to induce neurodegeneration and memory impairment $(45,46)$. Decreased zinc carrier gluconate may affect zinc balance in the brain. Twelve group D compounds (amino acids, nucleosides, choline, and carnitine) are plasma-enriched compounds and may underpin the actions of other metabolites for supply and degradation. Last, the two group $\mathrm{E}$ compounds, caffeine and dimethyl-xanthine, also decrease in dementia. Caffeine's beneficial effect on dementia was reported (47). Caffeine, an antioxidant purine, and its derivative, dimethylxanthine (highly correlated to each other) decline greatly in dementia subjects. Their relationship to dementia has been investigated, and caffeine may be a possible protectant against cognitive decline $(28,48)$, because of its antioxidative purine activity. Caffeine is an antagonist of adenosine (49), consistent with the present finding that adenosine belongs to group A compounds. Caffeine and dimethyl-xanthine were correlated with trimethyltryptophan. Consistently, the interaction between caffeine and tryptophan in the brain is known (50).

Mitochondrial dysfunction has been reported in dementia subjects $(51,52)$, which may explain the reduced level of ATP documented in this study in dementia subjects. ATP was clearly diminished in dementia, whereas the level was average or above in HE subjects. Our previous reports indicated that more than $98 \%$ of ATP in whole blood derives from RBCs $(14,53)$. Therefore, a decline in ATP level in dementia subjects is thought to occur mainly in RBCs. However, it may be necessary to verify whether ATP in plasma is actually reduced. In correlation analysis, ATP 


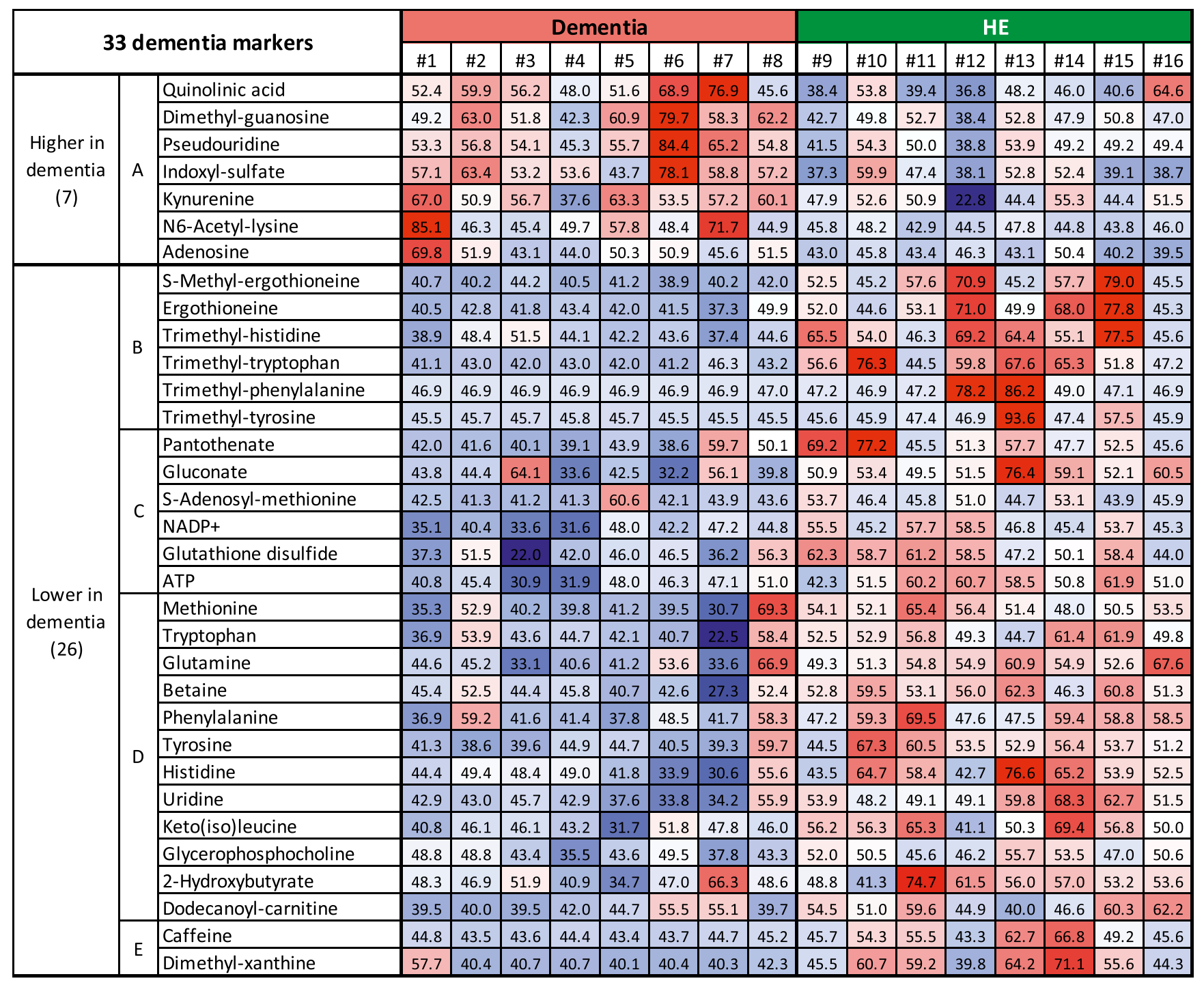

Fig. 4. Construction of a heatmap using abundance data of 33 compounds for each subject. Standardized scores ( $T$ scores) are represented by colors. Average value (50), white; values above average, pale red to deep red; values below average, pale blue to deep blue. Seven compounds that increased in dementia patients tended to be red in dementia whereas they tended to be white to blue in HE subjects. For the 26 remaining compounds, colors are reversed in dementia and HE subjects.

concentrations were positively correlated with 10 compounds in subgroups B to D (Fig. 3) so that the level of ATP may affect or be affected by concentrations of many metabolites, including oxidoreductive compounds, such as GSH, betaine, ET, and $S$-methylET, and amino acids, such as glutamine and tryptophan. Thus, it is quite possible that ATP might assist antioxidation in the brain via many metabolites present in plasma and RBCs so that it may contribute to brain activities against dementia. $\mathrm{NADP}^{+}$and GSSG may be synergistic in maintaining the level of ATP and ET (54). Hence, oxidoreductive $\mathrm{NADP}^{+}$, antioxidative GSH, and presumably neuroprotective trimethylated ammonium compounds may all function together to sustain brain mitochondrial ATP production level against dementia. Note that glycerophosphocholine and dodecanoyl-carnitine, which also contain a trimethyl-ammonium moiety, belong to group D and may also enhance mitochondrial function.

Nine compounds possessing trimethylated ammonium ions (Table 1 and SI Appendix, Fig. S2) are amphipathic compounds (possessing both hydrophilic and lipophilic properties) and form the basis of lipid polymorphism. All of them showed a sharp decline in abundance in dementia subjects. Distribution of ET in the brain has been demonstrated in various mammalian species $(55,56)$ and humans $(57)$. The cause of its decline might be due to the rise of ROS (reactive oxygen species) in dementia patient brains. These amphipathic compounds may have similar roles, such as forming a higher-ordered, assembled structure. In addition, these compounds are abundant. They might act as major neuroprotectants or antioxidants in the brain, and their levels are sensitive to both antioxidants and ROS. In addition, membrane defects have been observed in dementia patient brains that degrade glycerophosphocholine $(58,59)$. Alternatively, amphipathic moieties might deliver pertinent compounds of nanomolar concentrations to the CNS (60). An advantage of metabolomic analysis over proteomic and genomic studies exists in the search for drugs. As these metabolites exist in human body fluids, they may be employed for studies of drug therapy. In the present study, 33 metabolites are obvious targets for future study, the majority of which have not been studied at all relative to their clinical potential. 


\section{Materials and Methods}

Participants. Eight dementia patients, hospitalized at the National Hospital Organization Ryukyu Hospital, in Kin-town, Okinawa, participated as subjects in this study (SI Appendix, Table S1). They were judged carefully by doctors at Ryukyu Hospital to understand the study objectives and contents, privacy protection, and free choice of participation, and were selected. In addition, eight young and eight elderly healthy volunteers, who live in Onna-village, Okinawa, participated as volunteers. Blood samples for LC-MS measurements were collected between 2017 and 2018.

Diagnosis of subjects. Patients were diagnosed based on the Diagnostic and Statistical Manual of Mental Disorders, fourth edition (DSM-4) (61). Careful interviews on impairment of activities of daily living were performed to rule out delirium or other psychiatric diseases. Blood tests and interviews of patients ruled out the possibility of other systemic diseases. To assess cognitive ability and pathological changes, cognitive tests (HDS-R, MMSE, COGNISTAT), MRI for hippocampal atrophy for all patients, and voxel-based specific regional analysis system for Alzheimer's disease (VSRAD) for some patients were collected (SI Appendix, Table S1). Both the revised Hasegawa's Dementia Scale (HDS-R) (62) and the Mini-Mental State Examination (MMSE) $(63,64)$ have a maximum of 30 points, and cutoff values are 20/21 and 23/34, respectively. Dementia patients were below the cutoff values for each test, while HE people who agreed to the test were almost perfect ( 28 to 30 ). The results of the Japanese version of the neurobehavioral cognitive status examination, COGNISTAT (65), and the voxel-based specific regional analysis system for Alzheimer's disease, VSRAD (66), were done supplementally. The $Z$ score of VSRAD indicates the degree of atrophy of the middle temporal area, including the hippocampus (67). These clinical data were collected before January 2018 at Ryukyu Hospital. Information about patient medications is shown in $\mathrm{SI}$ Appendix, Table S4.

Ethics statement. Written informed consent was obtained from all participants in accordance with the Declaration of Helsinki. Consent of patient spouses or guardians was also obtained, in addition to that of the patients themselves. All experiments were performed in compliance with relevant Japanese laws and institutional guidelines. All protocols were approved by the Ethics Committee on Human Research of Ryukyu Hospital and by the Human Subjects Research Review Committee of the Okinawa Institute of Science and Technology Graduate University.

Chemicals and reagents. Standards for metabolite identification were purchased from commercial sources, as described previously $(14,15,53,68)$. LC MS-grade acetonitrile, methanol, and ultrapure water were obtained from FUJIFILM Wako Pure Chemical.

Blood sample preparation. Metabolomic samples were prepared as described previously (14). Briefly, venous blood samples were collected in heparinized tubes before breakfast. Subjects were asked to ensure at least $8 \mathrm{~h}$ of fasting prior to sampling. During fasting, subjects took water freely. Immediately, $0.2 \mathrm{~mL}$ of blood was quenched in $1.8 \mathrm{~mL}$ of $55 \%$ methanol at $-40{ }^{\circ} \mathrm{C}$. Ten nanomoles each of Hepes and Pipes was added to each sample to serve as

1. J. L. Cummings et al., The Neuropsychiatric Inventory: Comprehensive assessment of psychopathology in dementia. Neurology 44, 2308-2314 (1994).

2. Z. Arvanitakis, R. C. Shah, D. A. Bennett, Diagnosis and management of dementia: Review. JAMA 322, 1589-1599 (2019).

3. K. A. Jellinger, J. Attems, Neuropathological evaluation of mixed dementia. J. Neurol. Sci. 257, 80-87 (2007).

4. J. A. Schneider, Z. Arvanitakis, S. E. Leurgans, D. A. Bennett, The neuropathology of probable Alzheimer disease and mild cognitive impairment. Ann. Neurol. 66, 200-208 (2009).

5. S. K. Pope, V. M. Shue, C. Beck, Will a healthy lifestyle help prevent Alzheimer's disease? Annu. Rev. Public Health 24, 111-132 (2003).

6. M. Valenzuela, P. Sachdev, Can cognitive exercise prevent the onset of dementia? Systematic review of randomized clinical trials with longitudinal follow-up. Am J. Geriatr. Psychiatry 17, 179-187 (2009).

7. J. E. Ahlskog, Y. E. Geda, N. R. Graff-Radford, R. C. Petersen, Physical exercise as a preventive or disease-modifying treatment of dementia and brain aging. Mayo Clin. Proc. 86, 876-884 (2011).

8. J. Cummings et al., Drug development in Alzheimer's disease: The path to 2025 . Alzheimers Res. Ther. 8, 39 (2016).

9. G. Livingston et al., Dementia prevention, intervention, and care: 2020 report of the Lancet Commission. Lancet 396, 413-446 (2020).

10. A. Wimo, L. Jönsson, J. Bond, M. Prince, B. Winblad; Alzheimer Disease International, The worldwide economic impact of dementia 2010. Alzheimers Dement. 9, 1-11.e3 (2013). standards, After brief vortexing, samples were transferred to Amicon Ultra $10-k D a$ cutoff filters (Millipore) to remove proteins and cellular debris. After sample concentration by vacuum evaporation, each sample was resuspended in $40 \mu \mathrm{L}$ of $50 \%$ acetonitrile, and $1 \mu \mathrm{L}$ was used for each injection into the LCMS system, as described.

LC-MS analysis. Nontargeted LC-MS conditions were as described previously $(14,16)$. Briefly, LC-MS data were obtained using an UltiMate 3000 DGP3600RS high-performance liquid chromatography system (Thermo Fisher Scientific) coupled to an LTQ Orbitrap mass spectrometer (Thermo Fisher Scientific). LC separation was performed on a ZIC-pHILIC column (Merck SeQuant $150 \times 2.1 \mathrm{~mm}, 5-\mu \mathrm{m}$ particle size). Acetonitrile (A) and $10 \mathrm{mM}$ ammonium carbonate buffer ( $\mathrm{pH}$ 9.3) (B) were used as the mobile phase, with a linear gradient from 80 to $20 \%$ A over $30 \mathrm{~min}$, at a flow rate of $100 \mu \mathrm{L} / \mathrm{min}$. The mass spectrometer was operated in full-scan mode with a 100 to $1,000 \mathrm{~m} / \mathrm{z}$ scan rate and automatic data-dependent MS/MS fragmentation scans.

LC-MS data processing and peak characteristics. Peak areas of metabolites of interest were measured using MZmine 2 software (69). Data analytical procedures and parameters have been described previously (15). We analyzed 124 blood metabolites that were confirmed using standards or MS/MS analysis (Dataset S1). According to their peak areas, metabolite abundances were classified into three groups $(\mathrm{H}, \mathrm{M}$, and $\mathrm{L})$. $\mathrm{H}$ denotes compounds with high peak areas ( $>10^{8} \mathrm{AU}$; arbitrary unit), $\mathrm{M}$ denotes compounds with medium peak areas $\left(10^{7}\right.$ to $\left.10^{8} \mathrm{AU}\right)$, and $\mathrm{L}$ denotes compounds with low peak areas ( $\left.<10^{7} \mathrm{AU}\right)$ (SI Appendix, Table S2).

Statistical analysis. Peak data processed with MZmine 2 were exported into spreadsheet format and analyzed with R statistical software (http://www.rproject.org). Statistical analysis was performed using the Mann-Whitney $U$ test. Statistical significance was established at $P<0.05$. $Q$ values were calculated using the Benjamini-Hochberg method. The plot of PCs was generated with SIMCA-P+ software (Umetrics). Heatmaps represent standardized abundance data for each metabolite. $T$ scores were calculated from the following formula: $T$ score $=$ [(sample peak area - average of population peak area) $\times$ $10 /$ SD of population peak area] +50 . Therefore, the mean and SD are 50 and 10 , respectively.

Data Availability. Raw LC-MS data in mzML format reported in this article are accessible via the MetaboLights repository (https://www.ebi.ac.uk/metabolights). Data for the 24 subjects are available under accession no. MTBLS2109. All data are included in the manuscript and/or supporting information.

ACKNOWLEDGMENTS. We thank Ryukyu Hospital and the volunteers of Onna-village, without whom the study would not have been possible. We are grateful to Ms. Hiromi Karimata for excellent assistance in coordinating clinical data and documents. We thank Ms. Lisa Uehara, Dr. Michiko Suma, Ms. Ayaka Mori, and Ms. Yuria Tahara for providing excellent technical assistance, Ms. Kaori Serakaki for illustration (SI Appendix, Fig. S5), Dr. Reiko Sugiura for discussions on clinical data, and Dr. Steven D. Aird for editing the manuscript. We are greatly indebted to generous support from the Okinawa Institute of Science and Technology Graduate University and its Proof of Concept Program fund.

11. K. A. Stringer et al., Whole blood reveals more metabolic detail of the human metabolome than serum as measured by ${ }^{1} \mathrm{H}-\mathrm{NMR}$ spectroscopy: Implications for sepsis metabolomics. Shock 44, 200-208 (2015).

12. G. A. Nagana Gowda, D. Raftery, Whole blood metabolomics by ${ }^{1} \mathrm{H}$ NMR spectroscopy provides a new opportunity to evaluate coenzymes and antioxidants. Anal. Chem. 89 4620-4627 (2017)

13. H. Kondoh, M. Kameda, M. Yanagida, Whole blood metabolomics in aging research Int. J. Mol. Sci. 22, 175 (2020).

14. R. Chaleckis, I. Murakami, J. Takada, H. Kondoh, M. Yanagida, Individual variability in human blood metabolites identifies age-related differences. Proc. Natl. Acad. Sci. U.S.A. 113, 4252-4259 (2016)

15. T. Teruya, R. Chaleckis, J. Takada, M. Yanagida, H. Kondoh, Diverse metabolic reac tions activated during 58 -hr fasting are revealed by non-targeted metabolomic analysis of human blood. Sci. Rep. 9, 854 (2019)

16. M. Kameda, T. Teruya, M. Yanagida, H. Kondoh, Frailty markers comprise blood metabolites involved in antioxidation, cognition, and mobility. Proc. Natl. Acad. Sci. U.S.A. 117, 9483-9489 (2020)

17. I. K. Cheah, L. Feng, R. M. Y. Tang, K. H. C. Lim, B. Halliwell, Ergothioneine levels in an elderly population decrease with age and incidence of cognitive decline; a risk factor for neurodegeneration? Biochem. Biophys. Res. Commun. 478, 162-167 (2016).

18. E. Gulaj, K. Pawlak, B. Bien, D. Pawlak, Kynurenine and its metabolites in Alzheimer's disease patients. Adv. Med. Sci. 55, 204-211 (2010). 
19. R. González-Domínguez, T. García-Barrera, J. L. Gómez-Ariza, Metabolite profiling for the identification of altered metabolic pathways in Alzheimer's disease. J. Pharm. Biomed. Anal. 107, 75-81 (2015).

20. R. Vanholder et al.; European Uremic Toxin Work Group (EUTox), Review on uremic toxins: Classification, concentration, and interindividual variability. Kidney Int. 63, 1934-1943 (2003).

21. S. P. Guiraud et al., High-throughput and simultaneous quantitative analysis of homocysteine-methionine cycle metabolites and co-factors in blood plasma and cerebrospinal fluid by isotope dilution LC-MS/MS. Anal. Bioanal. Chem. 409, 295-305 (2017).

22. S. Ramachandran, P. Fontanille, A. Pandey, C. Larroche, Gluconic acid: Properties, applications and microbial production. Food Technol. Biotechnol. 44, 185-195 (2006).

23. I. Martínez de Toda, L. Miguélez, C. Vida, E. Carro, M. De la Fuente, Altered redox state in whole blood cells from patients with mild cognitive impairment and Alzheimer's disease. J. Alzheimers Dis. 71, 153-163 (2019).

24. A. Cristofano et al., Serum levels of acyl-carnitines along the continuum from normal to Alzheimer's dementia. PLoS One 11, e0155694 (2016).

25. R. González-Domínguez, T. García-Barrera, J. L. Gómez-Ariza, Using direct infusion mass spectrometry for serum metabolomics in Alzheimer's disease. Anal. Bioanal. Chem. 406, 7137-7148 (2014).

26. M. Mapstone et al., Plasma phospholipids identify antecedent memory impairment in older adults. Nat. Med. 20, 415-418 (2014).

27. M. G. Olde Rikkert et al., Differences in nutritional status between very mild Alz heimer's disease patients and healthy controls. J. Alzheimers Dis. 41, 261-271 (2014).

28. C. Cao et al., High blood caffeine levels in MCl linked to lack of progression to dementia. J. Alzheimers Dis. 30, 559-572 (2012).

29. M. Ozawa, K. Honda, I. Nakai, A. Kishida, A. Ohsaki, Hypaphorine, an indole alkaloid from Erythrina velutina, induced sleep on normal mice. Bioorg. Med. Chem. Lett. 18, 3992-3994 (2008)

30. C. A. Carollo et al., Fungal tyrosine betaine, a novel secondary metabolite from conidia of entomopathogenic Metarhizium spp. fungi. Fungal Biol. 114, 473-480 (2010).

31. T. W. Stone, G. M. Mackay, C. M. Forrest, C. J. Clark, L. G. Darlington, Tryptophan metabolites and brain disorders. Clin. Chem. Lab. Med. 41, 852-859 (2003).

32. S. Adesso et al., Indoxyl sulfate affects glial function increasing oxidative stress and neuroinflammation in chronic kidney disease: Interaction between astrocytes and microglia. Front. Pharmacol. 8, 370 (2017).

33. H. Iwabata, M. Yoshida, Y. Komatsu, Proteomic analysis of organ-specific posttranslational lysine-acetylation and -methylation in mice by use of anti-acetyllysine and -methyllysine mouse monoclonal antibodies. Proteomics 5, 4653-4664 (2005).

34. G. Sander, H. Topp, G. Heller-Schöch, J. Wieland, G. Schöch, Ribonucleic acid turnover in man: RNA catabolites in urine as measure for the metabolism of each of the three major species of RNA. Clin. Sci. (Lond.) 71, 367-374 (1986).

35. S. H. Lee, I. Kim, B. C. Chung, Increased urinary level of oxidized nucleosides in patients with mild-to-moderate Alzheimer's disease. Clin. Biochem. 40, 936-938 (2007)

36. F. Moroni, Tryptophan metabolism and brain function: Focus on kynurenine and other indole metabolites. Eur. J. Pharmacol. 375, 87-100 (1999).

37. J. P. Ruddick et al., Tryptophan metabolism in the central nervous system: Medical implications. Expert Rev. Mol. Med. 8, 1-27 (2006).

38. A. Rahman, The role of adenosine in Alzheimer's disease. Curr. Neuropharmacol. 7 207-216 (2009)

39. H. A. Mutsaers et al., Uremic toxins inhibit renal metabolic capacity through interference with glucuronidation and mitochondrial respiration. Biochim. Biophys. Acta 1832, 142-150 (2013).

40. G. J. Guillemin, B. J. Brew, Implications of the kynurenine pathway and quinolinic acid in Alzheimer's disease. Redox Rep. 7, 199-206 (2002).

41. T. J. Cohen, D. Friedmann, A. W. Hwang, R. Marmorstein, V. M. Lee, The microtubuleassociated tau protein has intrinsic acetyltransferase activity. Nat. Struct. Mol. Biol. 20, 756-762 (2013).

42. F. Zhang et al., Posttranslational modifications of $\alpha$-tubulin in Alzheimer disease. Transl. Neurodegener. 4, 9 (2015).

43. M. P. Cuajungco, G. J. Lees, Zinc and Alzheimer's disease: Is there a direct link? Brain Res. Brain Res. Rev. 23, 219-236 (1997).
44. T. Kambe, T. Tsuji, A. Hashimoto, N. Itsumura, The physiological, biochemical, and molecular roles of zinc transporters in zinc homeostasis and metabolism. Physiol. Rev. 95, 749-784 (2015)

45. M. A. Lovell, J. D. Robertson, W. J. Teesdale, J. L. Campbell, W. R. Markesbery, Copper, iron and zinc in Alzheimer's disease senile plaques. J. Neurol. Sci. 158, 47-52 (1998).

46. N. T. Watt, I. J. Whitehouse, N. M. Hooper, The role of zinc in Alzheimer's disease. Int. J. Alzheimers Dis. 2011, 971021 (2010).

47. S. C. Yenisetti, Muralidhara, Beneficial role of coffee and caffeine in neurodegenerative diseases: A minireview. AIMS Public Health 3, 407-422 (2016).

48. M. Kolahdouzan, M. J. Hamadeh, The neuroprotective effects of caffeine in neurodegenerative diseases. CNS Neurosci. Ther. 23, 272-290 (2017).

49. J. A. Ribeiro, A. M. Sebastião, Caffeine and adenosine. J. Alzheimers Dis. 20 (suppl. 1), S3-S15 (2010).

50. M. H. Fernstrom, C. W. Bazil, J. D. Fernstrom, Caffeine injection raises brain tryptophan level, but does not stimulate the rate of serotonin synthesis in rat brain. Life Sci. 35, 1241-1247 (1984)

51. C. Cadonic, M. G. Sabbir, B. C. Albensi, Mechanisms of mitochondrial dysfunction in Alzheimer's disease. Mol. Neurobiol. 53, 6078-6090 (2016).

52. D. A. Butterfield, B. Halliwell, Oxidative stress, dysfunctional glucose metabolism and Alzheimer disease. Nat. Rev. Neurosci. 20, 148-160 (2019).

53. R. Chaleckis et al., Unexpected similarities between the Schizosaccharomyces and human blood metabolomes, and novel human metabolites. Mol. Biosyst. 10, 2538-2551 (2014).

54. Y. Dong, S. Sameni, M. A. Digman, G. J. Brewer, Reversibility of age-related oxidized free NADH redox states in Alzheimer's disease neurons by imposed external Cys/CySS redox shifts. Sci. Rep. 9, 11274 (2019).

55. J. Crossland, J. Mitchell, G. N. Woodruff, The presence of ergothioneine in the central nervous system and its probable identity with the cerebellar factor. J. Physiol. 182, 427-438 (1966).

56. N. Nakamichi et al., Food-derived hydrophilic antioxidant ergothioneine is distributed to the brain and exerts antidepressant effect in mice. Brain Behav. 6, e00477 (2016).

57. S. F. Graham, O. P. Chevallier, P. Kumar, O. Türko Gcaron Lu, R. O. Bahado-Singh, Metabolomic profiling of brain from infants who died from sudden infant death syndrome reveals novel predictive biomarkers. J. Perinatol. 37, 91-97 (2017).

58. R. M. Nitsch et al., Evidence for a membrane defect in Alzheimer disease brain. Proc. Natl. Acad. Sci. U.S.A. 89, 1671-1675 (1992).

59. A. Walter et al., Glycerophosphocholine is elevated in cerebrospinal fluid of Alzheimer patients. Neurobiol. Aging 25, 1299-1303 (2004).

60. S. Guerrero et al., Improving the brain delivery of gold nanoparticles by conjugation with an amphipathic peptide. Nanomedicine (Lond.) 5, 897-913 (2010).

61. American Psychiatric Association, Diagnostic and Statistical Manual of Mental Disorders (American Psychiatric Association, Washington, DC, ed. 4, 1994).

62. Y. Imai, K. Hasegawa, The revised Hasegawa's Dementia Scale (HDS-R)-Evaluation of its usefulness as a screening test for dementia. J. Hong Kong College Psychiatry 4, 20-24 (1994).

63. M. F. Folstein, S. E. Folstein, P. R. McHugh, "Mini-mental state": A practical method for grading the cognitive state of patients for the clinician. J. Psychiatr. Res. 12, 189-198 (1975)

64. Y. Ideno, M. Takayama, K. Hayashi, H. Takagi, Y. Sugai, Evaluation of a Japanese version of the Mini-Mental State Examination in elderly persons. Geriatr. Gerontol. Int. 12, 310-316 (2012).

65. R. J. Kiernan, J. Mueller, J. W. Langston, C. Van Dyke, The Neurobehavioral Cognitive Status Examination: A brief but quantitative approach to cognitive assessment. Ann. Intern. Med. 107, 481-485 (1987).

66. Y. Hirata et al., Voxel-based morphometry to discriminate early Alzheimer's disease from controls. Neurosci. Lett. 382, 269-274 (2005).

67. G. Oshikubo et al., Utility of VSRAD for diagnosing Alzheimer's disease in patients screened for dementia. J. Int. Med. Res. 48, 300060520917270 (2020).

68. T. Pluskal, T. Nakamura, A. Villar-Briones, M. Yanagida, Metabolic profiling of the fission yeast $S$. pombe: Quantification of compounds under different temperatures and genetic perturbation. Mol. Biosyst. 6, 182-198 (2010).

69. T. Pluskal, S. Castillo, A. Villar-Briones, M. Oresic, MZmine 2: Modular framework for processing, visualizing, and analyzing mass spectrometry-based molecular profile data. BMC Bioinformatics 11, 395 (2010).
Teruya et al.

Whole-blood metabolomics of dementia patients reveal classes of disease-linked metabolites 